\title{
Analytical Capability of a High-Time Resolution-Multiple Collector-ICPMS (HTR-MC-ICPMS) system for Geochemical and Cosmochemical Studies
}

\author{
TAKAFUMI HIRATA ${ }^{1}$, ANDY BURROWS ${ }^{2}$ AND PHIL \\ FREEDMAN $^{2}$ \\ ${ }^{1}$ The University of Tokyo \\ ${ }^{2} \mathrm{Nu}$ Instruments \\ Presenting Author: hrt1@eqchem.s.u-tokyo.ac.jp
}

We have developed a High Time Resolution ion counting system on our Multi-Collector ICP-MS instrument, which allows for the simultaneous data acquisition of five isotopes with $10 \mu \mathrm{s}$ time resolution. This permits the quantitative analysis of transient signals (e.g., $<0.6 \mathrm{~ms}$ ) produced either by single-shot laser ablation or by introduction of single nanoparticles. The analytical capabilities of the system were demonstrated with two geochemical applications.

The first application focused on age determination from thin surface-layer $(<1 \mu \mathrm{m})$ of minerals, which provides key information on the thermal evolution sequences of rocks. The major problem associated with age determination from transient signals is that the resulting $\mathrm{U}-\mathrm{Pb}$ isotopic data can be erroneous. This is due to the under estimation of the counting loss of $\mathrm{U}-\mathrm{Pb}$ signals through the moderation of the signal intensity from neighbouring data acquisition time slices. With the correction of the counting loss based on data obtained from a short dwell time $(0.02 \mathrm{~ms})$, the resulting $206 \mathrm{~Pb} / 238 \mathrm{U}$ ratios obtained from a zircon and a glass standard were in good agreement with literature values within analytical uncertainties (2-3\%).

The elemental/isotopic analysis of nanoparticles (NPs) was chosen as a second application. Although size distribution analysis of NPs can be achieved in a short time period, analytical restrictions still remain, since the system is not capable of monitoring elemental composition or isotope ratios of the constituent elements in NPs. With the HTR-MC-ICPMS technique, the simultaneous detection of multiple elements or isotopes was achieved without lowering the time efficiencies of the signal integrations. The Pt and Os isotopic ratios for several naturally occurring minerals we obtained with this method were in good agreement with literature values. Ion counters with a wide dynamic range are also key to obtain reliable elemental/isotopic ratio data from transient signals. As such, the Daly detectors used, which accept ion beams of up to $>10 \mathrm{MCps}$, were perfectly suited for this type measurements.

High Time Resolution ion counters, coupled with Daly detectors therefore have the potential to become a major analytical tool for the precise elemental/isotopic analysis of trace components from transient signal with multi-collector instruments. 